PROCEEDINGS OF THE

AMERICAN MATHEMATICAL SOCIETY

Volume 128, Number 9, Pages 2585-2591

S 0002-9939(00)05708-7

Article electronically published on February 28, 2000

\title{
COMPACT SCHUR MULTIPLIERS
}

\author{
MILAN HLADNIK \\ (Communicated by David R. Larson)
}

\begin{abstract}
Compact Schur multipliers on the algebra $B(\mathcal{H})$ of all bounded linear operators on an infinite-dimensional separable complex Hilbert space $\mathcal{H}$ will be identified as the elements of the Haagerup tensor product $c_{0} \otimes^{h} c_{0}$ (the completion of $c_{0} \otimes c_{0}$ in the Haagerup norm). Other ideals of Schur multipliers related to compact operators will also be characterized.
\end{abstract}

\section{INTRODUCTION}

Let $\mathcal{H}$ be an infinite-dimensional separable complex Hilbert space with a fixed orthonormal basis $\left\{\epsilon_{i}\right\}_{i=1}^{\infty}$, and let $B=B(\mathcal{H})$ be the algebra of all bounded linear operators on $\mathcal{H}$. We shall identify every $\xi \in \mathcal{H}$ by the (infinite) column and every $x \in B$ by the (infinite) matrix according to the given basis and often simply write $\xi=\left(\xi_{i}\right)$ and $x=\left(x_{i j}\right)$. For a long time it has been well known that the Schur or entrywise product $x * y=\left(x_{i j} y_{i j}\right)$ is an internal operation in $B$ (see [10]) which turns $B$ into a semisimple commutative Banach algebra (with the usual operator norm $\|\cdot\|$ and without unity). We shall refer to this algebra as the Schur algebra and write $B_{*}$ instead of $B$ in order to stress the new product in $B$. Some of its Banach algebra properties including the construction of its maximal ideal space were determined by Q.F. Stout [9] in a slightly more general situation.

By a Schur multiplier we shall understand any map $m: B_{*} \rightarrow B_{*}$ such that $m(x * y)=m(x) * y$ for every $x, y \in B_{*}$. It is known that every Schur multiplier is a continuous linear operator on $B_{*}$ and that $M_{*}=M\left(B_{*}\right)$, the set of all Schur multipliers, is a (maximal) commutative Banach subalgebra of $B\left(B_{*}\right)$, the algebra of all bounded linear operators on $B_{*}$ (see [7, Theorem 1.1.1). The norm is inherited from $B\left(B_{*}\right)$ and will be called the multiplier (or the Schur) norm and denoted by $\|\cdot\|_{m}$ to distinguish it from the norm $\|\cdot\|$ in the Schur algebra $B_{*}$. Since $\|x * y\| \leq\|x\|\|y\|$ holds for every $x, y \in B_{*}$ (see [10]) the algebra $B_{*}$ can be considered as a subalgebra in $M_{*}$, and we have $\|x\|_{m} \leq\|x\|$ for every $x \in B_{*}$. It is easy to verify that the two norms on $B_{*}$ are not equivalent and that $B_{*}$ is not a closed subalgebra in $M_{*}$. Thus, ${\overline{B_{*}}}^{m}$, the closure in the multiplier norm,

Received by the editors October 7, 1998.

2000 Mathematics Subject Classification. Primary 47B07, 47B49; Secondary 46M05, 47L20.

Key words and phrases. Schur multipliers, compact and weakly compact multipliers, Haagerup tensor products.

This work was supported in part by the Ministry of Science and Technology of Slovenia.

The author expresses his gratitude to Professor Bojan Magajna for a discussion concerning the Haagerup tensor product of $C^{*}$-algebras and for a careful reading of the first version of this paper. 
is strictly larger than $B_{*}$. We shall use the subscript $m$ also to distinguish the convergence in the multiplier norm from the convergence in the operator norm, e.g. $x_{n} \rightarrow_{m} x$ or $x=\lim _{m} x_{n}$ contrary to $x_{n} \rightarrow x$ or $x=\lim x_{n}$. From time to time we shall also need other types of convergence of multipliers. For instance, the convergence in the point norm topology on $B\left(B_{*}\right)$, i.e. $m_{n} \rightarrow_{p n} m$ if and only if $m_{n}(y) \rightarrow m(y)$ for every $y \in B_{*}$, or the convergence in the point weak operator topology on $B\left(B_{*}\right): m_{n} \rightarrow$ pwot $m$ if and only if $\left\langle m_{n}(y) \xi, \eta\right\rangle \rightarrow\langle m(y) \xi, \eta\rangle$ for every $y \in B_{*}$ and $\xi, \eta \in \mathcal{H}$. (Note that the point norm topology is sometimes called the strong operator topology. However, we shall not use this term to avoid confusion with the usual strong operator topology on $B(\mathcal{H})$.) A convenient reference for general theory of multipliers of (commutative) Banach algebras is [7].

If $e_{i j}$ is the usual matrix unit and $m \in M_{*}$ is any Schur multiplier, write $m_{i j}=$ $m\left(e_{i j}\right)_{i j}$ for every $i, j$. Then it is easy to deduce from the identity $m(x * y)=m(x) * y$ on $B_{*}$, that $m\left(e_{i j}\right)=m_{i j} e_{i j}$ and that $m(x)=\left(m_{i j} x_{i j}\right)$ if $x=\left(x_{i j}\right) \in B_{*}$. This may be interpreted as the Schur product and, hence, written as $m * x$ (in the sequel we shall always write $m * x$ instead of $m(x))$. For example, in the case of the point norm convergence we have $m_{n} * y \rightarrow m * y$ for every $y \in B_{*}$. Moreover, the product in $M_{*}$, inherited from $B\left(B_{*}\right)$, can also be identified with the Schur product of the appropriate matrices, in accordance with the fact that $B_{*}$ is a subalgebra in $M_{*}$. For elementary properties of Schur multipliers regarded as infinite matrices and for some generalisations see [1].

There is still another useful interpretation of Schur multipliers. Namely, we can see them as precisely those elements in $B(B(\mathcal{H})$ ) which commute with every left or right multiplication by diagonal operators in $B(\mathcal{H})$ (in the given orthonormal basis). We make the following convention: if $\alpha=\left(a_{i}\right), \beta=\left(b_{j}\right)$ are two complex sequences (columns), let us agree to denote by $\alpha \otimes \beta$ the matrix $\left(a_{i} b_{j}\right)$.

Lemma 1. $M_{*}$ is equal to the algebra of all bimodule endomorphisms from $B$ to $B$ regarding $B=B(\mathcal{H})$ as a Banach bimodule over the commutative algebra $D$ of all diagonal operators in the given basis.

Proof. The statement of the lemma means precisely that $m \in M_{*}$ if and only if $m(a x b)=a m(x) b$ for every $x \in B_{*}$ and $a, b \in D$, the algebra of diagonal operators in $B(\mathcal{H})$. To see this let $\alpha=\left(a_{i}\right)$ and $\beta=\left(b_{j}\right)$ be bounded sequences (columns) consisting of diagonal entries of $a$ and $b$, respectively. Then, by the previous convention, we can write $a x b=\left(a_{i} b_{j} x_{i j}\right)=(\alpha \otimes \beta) * x$, so that $\alpha \otimes \beta \in M_{*}$. The only if direction of the above assertion is now obvious since $m(a x b)=m((\alpha \otimes \beta) * x)=(\alpha \otimes \beta) * m(x)=a m(x) b$, while the converse follows from $m(x)_{i j} e_{i j}=e_{i i} m(x) e_{j j}=m\left(e_{i i} x e_{j j}\right)=x_{i j} m\left(e_{i j}\right)=m_{i j} x_{i j} e_{i j}$ for each pair $i, j$ of indices.

In the sequel we are going to characterize the ideal of compact multipliers on $B_{*}$, first (in Section 2) as the multiplier norm closure of compact operators on $\mathcal{H}$, and then (in Section 3) as the Haagerup tensor product $c_{0} \otimes^{h} c_{0}$. Similarly, we identify the ideal of weakly compact multipliers with the point norm closure of compact operators and later also relate it with the tensor product of $c_{0}$ and $l_{\infty}$. Finally, the point weak operator closure of compact operators turns out to be the whole multiplier algebra $M_{*}$ and can be identified with the normal Haagerup tensor product $l_{\infty} \otimes^{\sigma h} l_{\infty}$. It follows then from the duality theory for the Haagerup tensor product that $M_{*}$ is the second dual of the ideal of all compact multipliers. 


\section{Compact multipliers}

The ideal $K(\mathcal{H})$ of all compact operators is not only (a closed) ideal of $B(\mathcal{H})$ with the usual product of operators but also a closed Schur ideal, i.e. an ideal in the Schur algebra $B_{*}$, closed in the operator norm of $B_{*}$ (see [9], Proposition 2.5). However, it is not a maximal proper ideal in $B_{*}$ as we shall see later. According to our previous notation we shall denote it as $K_{*}$.

Thus, we can consider multipliers on $K_{*}$, that is, bounded linear maps $m: K_{*} \rightarrow$ $K_{*}$ with the property $m(x * y)=m(x) * y$ for every $x, y \in K_{*}$. It is natural to denote the set of all multipliers on $K_{*}$ by $M\left(K_{*}\right)$. However, both kinds of multipliers are closely related.

Lemma 2. The second Banach adjoint of a multiplier $m \in M\left(K_{*}\right)$ is a Schur multiplier $m^{\prime \prime} \in M_{*}$ and for every $m^{\prime \prime} \in M_{*}$ its restriction $m=\left.m^{\prime \prime}\right|_{K_{*}}$ is a multiplier on $K_{*}$. Both multipliers have the same matrix $\left(m_{i j}\right)$.

Proof. We use the duality between $K_{*}=K$ and the trace class $T=K^{\prime}$ and the duality between $T$ and $B_{*}=B=T^{\prime}$ given by the bilinear form $(x, y) \mapsto$ $\operatorname{tr}(x y)$ where $\operatorname{tr}$ denotes the operator trace on $T$. For $m=\left(m_{i j}\right) \in M\left(K_{*}\right)$ its Banach adjoint $m^{\prime}$ on $T$ is equal to the operator with the transposed matrix: $m^{\prime}=$ $\left(m_{j i}\right)$. This easily follows from the identity $\operatorname{tr}((m * x) y)=\sum_{i} \sum_{j}\left(m_{i j} x_{i j}\right) y_{j i}=$ $\sum_{i} \sum_{j} x_{i j}\left(m_{j i}^{\prime} y_{j i}\right)=\operatorname{tr}\left(x\left(m^{\prime} * y\right)\right)$ valid for $x \in K_{*}$ and $y \in T$. In the same way the Banach adjoint of $m^{\prime}$ is $m^{\prime \prime}$ acting on $B_{*}$ via the matrix $\left(m_{i j}\right)$. Hence, $m^{\prime \prime}$ is a multiplier on $B_{*}$ which leaves $K_{*}$ invariant and $m=\left.m^{\prime \prime}\right|_{K_{*}}$.

By Lemma 2 we have $M\left(K_{*}\right)=\left.M_{*}\right|_{K_{*}}$. Since $K_{*}$ has an approximate identity $\left\{e_{n}\right\}$ where $e_{n}=\sum_{i, j=1}^{n} e_{i j}=\epsilon_{(n)} \otimes \epsilon_{(n)}$ and $\epsilon_{(n)}=(1,1, \ldots, 1,0, \ldots)^{\top}$ with the first $n$ entries equal to 1 for every $n$, it follows easily from $e_{n} * x \rightarrow x, x \in K_{*}$, that also $\left(m * e_{n}\right) * x \rightarrow m * x$ for every $m \in M_{*}$ and hence also for every $m \in M\left(K_{*}\right)$. Thus, we have $M\left(K_{*}\right) \subset \overline{K_{*}}$ (the closure in the point norm topology on $B\left(K_{*}\right)$ ). As the converse inclusion is obvious we have in fact the equality $M\left(K_{*}\right)=\overline{K_{*}}$ (see also 7], Theorem 1.1.6). Note that $\bar{K}_{*}$ must not be confused with ${\overline{K_{*}}}^{p n}$ (the closure in the point norm topology on $B\left(B_{*}\right)$ ) which is strictly smaller and which will play a substantial role in the sequel.

Using the same argument with the approximate identity as above one can show that $K_{*}$ is an ideal also in $M_{*}$, the algebra of all Schur multipliers on $B_{*}$. However, $K_{*}$ is not closed in $M_{*}$ in the multiplier norm as we can see by considering the sequence $m_{n}=x_{1} \oplus x_{2} \oplus \ldots \oplus x_{n}$ with $x_{n}=y_{n} / \sqrt{n}$ where $y_{n}$ is the $n \times n$-matrix with all entries equal to 1 . The sequence $\left(m_{n}\right)$ converges in $M_{*}$ but not in $B_{*}$. Moreover, its limit does not belong to $K_{*}$ because it is not a bounded operator on $\mathcal{H}$ at all. The closure ${\overline{K_{*}}}^{m}$ is a closed ideal in $M_{*}$. The same is true for ${\overline{K_{*}}}^{p n}$, the closure in the point norm topology, or for ${\overline{K_{*}}}^{\text {pwot }}$, the closure in the point weak operator topology. We clearly have $K_{*} \subset{\overline{K_{*}}}^{m} \subset{\overline{K_{*}}}^{p n} \subset{\overline{K_{*}}}^{\text {pwot }}$. We intend to show that these closures are different and to identify them with other known classes of multipliers.

Proposition 1. The ideal ${\overline{K_{*}}}^{m}$ is equal to the set of all compact Schur multipliers on the algebra $B_{*}$.

Proof. Since $K_{*}$ is the closure of matrices with finitely many non-zero entries (the left upper corners of matrices) and since the convergence in the norm $\|$.$\| implies$ 
the convergence in the norm $\|\cdot\|_{m}$, every element in ${\overline{K_{*}}}^{m}$ is a compact operator. Conversely, we already know that $e_{n} *(m * x) \rightarrow m * x$ for $x \in K_{*}$ and $m \in M_{*}$. If $m$ is a compact multiplier, then this convergence is uniform on the unit ball in $K_{*}$. Hence, we have $m \in{\overline{K_{*}}}^{m}$.

Proposition 2. The ideal ${\overline{K_{*}}}^{\text {pwot }}$ is equal to the set $M_{*}$ of all Schur multipliers on $B_{*}$.

Proof. Let $e_{n}=\epsilon_{(n)} \otimes \epsilon_{(n)}$ with $\epsilon_{(n)}=(1,1, \ldots, 1,0, \ldots)^{\top}$ as before. Denoting $\xi_{(n)}=\epsilon_{(n)} * \xi=\left(\xi_{1}, \xi_{2}, \ldots, \xi_{n}, 0, \ldots\right)^{\top}$ and $\eta_{(n)}=\epsilon_{(n)} * \eta=\left(\eta_{1}, \eta_{2}, \ldots, \eta_{n}, 0, \ldots\right)^{\top}$ for $\xi, \eta \in \mathcal{H}$ it is clear that $\xi_{(n)} \rightarrow \xi$ and $\eta_{(n)} \rightarrow \eta$ as $n \rightarrow \infty$. Then we have for every $m \in M_{*}, x \in B_{*}, \xi, \eta \in \mathcal{H}$ the convergence

$$
\begin{aligned}
\left\langle\left(\left(e_{n} * m\right) * x\right) \xi, \eta\right\rangle & =\left\langle(m * x)\left(\epsilon_{(n)} * \xi\right), \epsilon_{(n)} * \eta\right\rangle \\
& =\left\langle(m * x) \xi_{(n)}, \eta_{(n)}\right\rangle \rightarrow\langle(m * x) \xi, \eta\rangle .
\end{aligned}
$$

This means that $e_{n} * m \rightarrow$ pwot $m$.

Proposition 3. The ideal ${\overline{K_{*}}}^{\text {pn }}$ is equal to the set of all Schur multipliers which maps the algebra $B_{*}$ into $K_{*}$, i.e. $\overline{K_{*}}{ }^{n}=\left\{m \in M_{*} ; m * x \in K_{*}\right.$ for every $\left.x \in B_{*}\right\}$.

Proof. If $m \in{\overline{K_{*}}}^{p n}$, then $m * x=\lim \left(x_{n} * x\right)$ for every $x \in B_{*}$ and some sequence $x_{n} \in K_{*}$. Since $x_{n} * x \in K_{*}$, also $m * x \in K_{*}$. Conversely, if $m * x \in K_{*}$ for every $x \in B_{*}$, then with $\left\{e_{n}\right\}$, the usual approximate identity in $K_{*}$, we have $m * x=\lim \left((m * x) * e_{n}\right)=\lim \left(\left(m * e_{n}\right) * x\right)$ for every $x \in B_{*}$; hence, $m=$ $\lim _{p n}\left(m * e_{n}\right) \in{\overline{K_{*}}}^{p n}$.

Corollary 1. The ideal ${\overline{K_{*}}}^{p n}$ is exactly the set of all weakly compact Schur multipliers.

Proof. By [4], VI.4.2 and VI.4.8, a Schur multiplier $m=\left(\left.m\right|_{K_{*}}\right)^{\prime \prime}$ (see Lemma 2) is weakly compact if and only if $m\left(B_{*}\right) \subset K_{*}$. By Proposition 3 this is the case if and only if $m \in{\overline{K_{*}}}^{p n}$.

Examples. The following examples are constructed to distinguish among the ideals $K_{*},{\overline{K_{*}}}^{m},{\overline{K_{*}}}^{p n}$, and ${\overline{K_{*}}}^{p w o t}$ (even if the intersection with $B_{*}$ is considered).

1. We have already seen that $K_{*} \neq{\overline{K_{*}}}^{m}$. The sequence $m_{n}=x_{1} \oplus x_{2} \oplus \ldots \oplus x_{n}$, with $x_{n}=y_{n} / \sqrt{n}$ where $y_{n}$ is now the $n \times n$-matrix with 1 in the first column and 0 otherwise, converges in the strong operator topology on $B_{*}$ and also in the multiplier norm on $B_{*}$. Hence, its limit $m$ belongs to $B_{*} \cap{\overline{K_{*}}}^{m}$. However, it does not belong to $K_{*}$ since $m^{*} m=x_{1}^{*} x_{1} \oplus x_{2}^{*} x_{2} \oplus x_{3}^{*} x_{3} \oplus \ldots$, where * denotes the Hilbert space adjoint, is a projection with infinite-dimensional range and, hence, not in $K_{*}$. Thus, $B_{*} \cap{\overline{K_{*}}}^{m}$ is an ideal in $B_{*}$, strictly larger than $K_{*}$. It is easy to see that it is closed in the norm $\|\cdot\|$ of the algebra $B_{*}$.

2. Let $\alpha=(1,1 / 2,1 / 3, \ldots)^{\top}$ and $\epsilon_{(n)}=(1,1, \ldots, 1,0, \ldots)^{\top}$ as before. Then $\alpha \otimes \epsilon_{(n)}$ is a matrix with the first $n$ columns equal to $\alpha$ and zero otherwise. Denoting by $a$ and $e_{(n)}$ the diagonal matrix with the diagonal entries from $\alpha$ and $\epsilon_{(n)}$, respectively, we have $\left(\alpha \otimes \epsilon_{(n)}\right) * x=\operatorname{axe}_{(n)}$ for every $x \in B_{*}$. Since $a$ is compact and since $e_{(n)}$ converges in the strong operator topology on $B_{*}$ to the identity operator, $\left(\alpha \otimes \epsilon_{(n)}\right) * x=\operatorname{axe}_{(n)}$ converges to $a x$ in the norm of $B_{*}$. Hence, the left multiplication by $a$ (having a matrix with all columns equal to $\alpha$ ) belongs to ${\overline{K_{*}}}^{p n}$. On 
the other hand, since all of its eigenspaces are of infinite dimension it is not compact, and, thus, not in ${\overline{K_{*}}}^{m}$ by Proposition 1 . However, we do not know whether $B_{*} \cap{\overline{K_{*}}}^{p n}$ is strictly larger than $B_{*} \cap{\overline{K_{*}}}^{m}$.

3. It is possible to show that $B_{*} \cap{\overline{K_{*}}}^{p n} \neq B_{*} \cap{\overline{K_{*}}}^{p w o t}$. Take for example the identity operator $i \in B_{*}$ which is the limit of the projections $x_{n}=e_{11}+e_{22}+\ldots+e_{n n}$ in the point weak operator topology but not in the point norm topology. Moreover, $i$ cannot belong to ${\overline{K_{*}}}^{p n}$. If it were so, then $i$ would be the limit in the point norm topology of a sequence $y_{n} \in K_{*}$ with only left upper corners of its matrices different from zero. But, $i=i * i \neq \lim y_{n} * i$ because $\left\|i-y_{n} * i\right\|=1$ for every $n$. By Proposition 2 we also know that $B_{*} \cap{\overline{K_{*}}}^{\text {pwot }}=B_{*}$.

\section{TENSOR PRODUCTS}

We relate now the above ideals to various kinds of Haagerup tensor products of $C^{*}$-algebra $c_{0}$ of all complex sequences converging to zero. First of all we may see the elements of the algebraic tensor product $l_{2} \otimes l_{2}$ as the matrix representation of finite-rank operators on $\mathcal{H}$, identifying $\sum_{k=1}^{n} \alpha_{k} \otimes \beta_{k}$ with the matrix $\left(\sum_{k=1}^{n} a_{k i} b_{k j}\right)$ if $\alpha_{k}=\left(a_{k i}\right)$ and $\beta_{k}=\left(b_{k j}\right)$ belong to $l_{2}$. In the same way we consider also elements in $c_{0} \otimes c_{0}$ and $l_{\infty} \otimes l_{\infty}$ as appropriate matrices.

Proposition 4. $B_{*} \cap\left(l_{\infty} \otimes l_{\infty}\right)=l_{2} \otimes l_{2}$.

Proof. Obviously, we have $l_{2} \otimes l_{2} \subset B_{*}$ and $l_{2} \otimes l_{2} \subset l_{\infty} \otimes l_{\infty}$. Conversely, let $\alpha_{k}, \beta_{k} \in l_{\infty}$ for $k=1,2, \ldots, n$ and let $\sum_{k=1}^{n} \alpha_{k} \otimes \beta_{k}$ be bounded as an operator in $B_{*}$. This means that $\sum_{i}\left|\sum_{k=1}^{n} a_{k i} \sum_{j} b_{k j} \xi_{j}\right|^{2}<\infty$ for every $\xi=\left(\xi_{j}\right) \in l_{2}(=\mathcal{H})$. Hence, $\sum_{i}\left|\sum_{k=1}^{n} a_{k i}\left\langle\xi, \beta_{k}\right\rangle\right|^{2}<\infty$ also for every $\xi \in l_{1}$ where $\left\langle\xi, \beta_{k}\right\rangle=\sum_{j} b_{k j} \xi_{j}$ for $k=1,2, \ldots, n$. Take $\xi \in l_{1}$ such that $\left\langle\xi, \beta_{k}\right\rangle=0$ for each $k \neq k_{0}$, and $\left\langle\xi, \beta_{k_{0}}\right\rangle=1$. Then $\sum_{i}\left|a_{k_{0} i}\right|^{2}<\infty$ and so $\alpha_{k_{0}} \in l_{2}$. Since $\sum_{k=1}^{n} \beta_{k} \otimes \alpha_{k}$ is also bounded, being the transpose of $\sum_{k=1}^{n} \alpha_{k} \otimes \beta_{k}$, we get similarly that $\beta_{k_{0}} \in l_{2}$ for any $k_{0}$. Thus, we have proved $\sum_{k=1}^{n} \alpha_{k} \otimes \beta_{k} \in l_{2} \otimes l_{2}$.

Corollary 2. $K_{*} \cap\left(c_{0} \otimes c_{0}\right)=l_{2} \otimes l_{2}$.

Proof. This follows from $l_{2} \otimes l_{2} \subset K_{*} \cap\left(c_{0} \otimes c_{0}\right) \subset B_{*} \cap\left(l_{\infty} \otimes l_{\infty}\right)=l_{2} \otimes l_{2}$.

There is a natural map $\theta$ from the algebraic tensor product $c_{0} \otimes c_{0}$ into the algebra of all bimodule endomorphisms of $B=B_{*}$ regarded as the Banach bimodule over the diagonal algebra $D$ (hence, to the algebra $M_{*}$ of all Schur multipliers on $B_{*}$ by Lemma 1). Namely, let $a_{k}$ and $b_{k}$ be diagonal operators (matrices) with the diagonal entries $a_{k i}$ from $\alpha_{k}$ and $b_{k j}$ from $\beta_{k}$, respectively, for every $k$. Then $\theta$ is given by $\theta\left(\sum_{k} \alpha_{k} \otimes \beta_{k}\right)=\sum_{k} a_{k} x b_{k}$. Recall also the definition of the Haagerup norm on the algebraic tensor product of two $C^{*}$-algebras (in this case both equal to $\left.c_{0}\right)$ :

$$
\|u\|_{h}=\inf \left\|\sum_{k} \alpha_{k} \alpha_{k}^{*}\right\|^{1 / 2}\left\|\sum_{k} \beta_{k}^{*} \beta_{k}\right\|^{1 / 2},
$$

where the infimum is taken over all representations of $u=\sum_{k} \alpha_{k} \otimes \beta_{k}$ as a finite sum of elementary tensors (see e.g. [2]). Then, by the injectivity of the Haagerup tensor product (see e.g. [2] or [8]) and by the isometric imbedding of $l_{\infty} \otimes^{h} l_{\infty}$, the completion in the Haagerup norm, into the algebra of all completely bounded bimodule endomorphisms (see e.g. [11], Theorem 4.3), the map $\theta$ is an isometry. But, further, since the algebra $D$, acting on $\mathcal{H}$, has a cyclic vector, it follows from 
11], Theorem 2.1, that the completely bounded norm of bimodule endomorphisms coincides with its operator norm, and, hence, $\theta$ can be extended to an isometry from $c_{0} \otimes{ }^{h} c_{0}$ (with the Haagerup norm) into $M_{*}$ equipped with the usual multiplier norm. For the sake of brevity we shall consider $\theta$ as an inclusion and the elements in $c_{0} \otimes c_{0}$ as Schur multipliers on $B_{*}$. In this way we shall simply identify $c_{0} \otimes^{h} c_{0}$ with ${\overline{c_{0} \otimes c_{0}}}^{m} \in M_{*}$.

By this convention we have the following tensor product characterization of the ideal of all compact Schur multipliers:

Proposition 5. ${\overline{K_{*}}}^{m}=c_{0} \otimes^{h} c_{0}$.

Proof. Since $K_{*}=\overline{l_{2} \otimes l_{2}}$ (in the operator norm), we have also ${\overline{K_{*}}}^{m}={\overline{l_{2} \otimes l_{2}}}^{m}$ (in the multiplier norm). From $l_{2} \otimes l_{2} \subset{\overline{c_{0} \otimes c_{0}}}^{m}$ we get ${\overline{K_{*}}}^{m}={\overline{l_{2} \otimes l_{2}}}^{m} \subset{\overline{c_{0} \otimes c_{0}}}^{m}=$ $c_{0} \otimes^{h} c_{0}$. On the other hand, note that $l_{2} \otimes l_{2}$ is dense in $c_{0} \otimes^{h} c_{0}$ in the Haagerup norm because $l_{2}$ is dense in $c_{0}$ in the supremum norm and because of the inequality

$$
\begin{aligned}
\left\|\alpha \otimes \beta-\alpha^{\prime} \otimes \beta^{\prime}\right\|_{h} & \leq\left\|\left(\alpha-\alpha^{\prime}\right) \otimes \beta\right\|_{h}+\left\|\alpha^{\prime} \otimes\left(\beta-\beta^{\prime}\right)\right\|_{h} \\
& =\left\|\alpha^{\prime}-\alpha\right\|\|\beta\|+\left\|\alpha^{\prime}\right\|\left\|\beta-\beta^{\prime}\right\|
\end{aligned}
$$

for $\alpha, \alpha^{\prime}, \beta, \beta^{\prime} \in c_{0}$. Thus, the equality holds in the above inclusion.

Note that $l_{\infty}$ is a commutative von Neumann algebra which can be represented on the Hilbert space $\mathcal{H}$ as the algebra $D$ of all diagonal operators (in a given basis). Its commutant in $B$ is the same: $D^{\prime}=D$. Hence, the so-called normal Haagerup tensor product $l_{\infty} \otimes^{\sigma h} l_{\infty}$, defined by Effros and Kishimoto in [6], may be identified isometrically with the algebra of all bimodule endomorphisms of $B=B_{*}$ regarded as the Banach bimodule over the diagonal algebra $D$ (see [6], Theorem 2.5). These bimodule endomorphisms are precisely Schur multipliers on $B_{*}$. Thus, Proposition 2 yields:

Proposition 6. ${\overline{K_{*}}}^{\text {pwot }}=l_{\infty} \otimes^{\sigma h} l_{\infty}$.

Corollary 3. $M_{*}$ is the second dual of ${\overline{K_{*}}}^{m}$.

Proof. This follows from Propositions 2 and 6 and the fact that the normal Haagerup tensor product $l_{\infty} \otimes^{\sigma h} l_{\infty}$ coincides with the second dual of $c_{0} \otimes^{h} c_{0}$ (the last assertion can be deduced for example from the general duality theory for the Haagerup tensor product in [5] and [3]).

It would be nice to express in the tensor product notation also the ideal ${\overline{K_{*}}}^{p n}$ of all weakly compact multipliers. Considering $l_{\infty} \otimes c_{0}$ and $c_{0} \otimes l_{\infty}$ as multipliers we get the following result:

Proposition 7. ${\overline{\bar{K}_{*}}}^{n}={\overline{l_{\infty} \otimes c_{0}+c_{0} \otimes l_{\infty}}}^{p n}$.

Proof. Note that $c_{0}$ is strongly dense in $l_{\infty}$, regarded as diagonal matrices. It follows that $l_{\infty} \otimes c_{0}$ and $c_{0} \otimes l_{\infty}$ are in the point norm closure of $c_{0} \otimes c_{0}$ because elements in $c_{0}$ give compact diagonal operators (see also the argumentation in Example 2). Hence, $l_{\infty} \otimes c_{0}+c_{0} \otimes l_{\infty} \subset{\overline{c_{0} \otimes c_{0}}}^{p n} \subset{\overline{K_{*}}}^{p n}$ and also ${\overline{l_{\infty} \otimes c_{0}+c_{0} \otimes l_{\infty}}}^{p n} \subset{\overline{K_{*}}}^{p n}$. On the other hand, ${\overline{K_{*}}}^{p n}={\overline{l_{2} \otimes l_{2}}}^{p n}$ and, thus, obviously, ${\overline{K_{*}}}^{p n} \subset{\overline{l_{\infty} \otimes c_{0}+c_{0} \otimes l_{\infty}}}^{p n}$. 


\section{REFERENCES}

[1] G. Bennett, Schur multipliers, Duke Math. J. 44 (1977), 603-639. MR 58:12490

[2] D.P. Blecher, V.I. Paulsen, Tensor products of operator spaces, J. Funct. Anal. 99 (1991), 262-292. MR 93d:46095

[3] D.P. Blecher, R.R. Smith, The dual of the Haagerup tensor product, J. London Math. Soc. (2) 45 (1992), 126-144. MR 93h:46078

[4] N. Dunford, J.T. Schwartz, Linear operators, Part I, Interscience. New York, 1958. MR 22:8302

[5] E.G. Effros, R. Exel, On multilinear double commutant theorems, London Math. Soc. Lect. Notes Ser. 135 (1988), 81-94. MR 91h:46104

[6] E.G. Effros, A. Kishimoto, Module maps and Hochschield-Johnson cohomology, Indiana Math. J. 36 (1987), 257-276. MR 89b:46068

[7] R. Larsen, An introduction to the theory of multipliers, Springer-Verlag, New York - Heidelberg - Berlin, 1971. MR 55:8695

[8] V.I. Paulsen, R.R. Smith, Multilinear maps and tensor norms on operator systems, J. Funct. Anal. 73 (1987), 258-276. MR 89m:46099

[9] Q.F. Stout, Schur multiplication on $\mathcal{B}\left(l_{p}, l_{q}\right)$, J. Operator Theory 5 (1981), 231-243. MR 82h:47028

[10] I. Schur, Bemerkung zur Theorie der beschrankten Bilinearformen mit unendlich vielen Veranderlichen, J. Reine Angew. Math. 140 (1911), 1 - 28.

[11] R.R. Smith, Completely bounded module maps and the Haagerup tensor product, J. Funct. Anal. 102 (1991), 156-175. MR 93a:46115

Department of Mathematics, University of Ljubljana, Jadranska 19, Ljubluana 1000 , SLOVENIA

E-mail address: Milan.Hladnik@fmf.uni-lj.si 